\title{
Validation and cultural adaptation of the Arabic versions of the Mini-Mental Status Examination - 2 and Mini-Cog test
}

This article was published in the following Dove Press journal:

Neuropsychiatric Disease and Treatment

14 March 2017

Number of times this article has been viewed

\author{
Mohammad Albanna ${ }^{1, *}$ \\ Arij Yehya ${ }^{2, *}$ \\ Abdalla Khairi' \\ Elnour Dafeeah' \\ Abdelsalam Elhadi ${ }^{3}$ \\ Lamia Rezgui ${ }^{4}$ \\ Shahada Al Kahlout ${ }^{4}$ \\ Adil Yousif ${ }^{5}$ \\ Basim Uthman ${ }^{6}$ \\ Hassen $\mathrm{Al}-\mathrm{Amin}^{2}$ \\ 'Psychiatry Department, Hamad \\ Medical Corporation, ${ }^{2}$ Psychiatry \\ Department, Weill Cornell Medicine - \\ Qatar, ${ }^{3}$ Primary Health Care \\ Corporation, ${ }^{4}$ Geriatrics Department, \\ Rumailah Hospital, Hamad Medical \\ Corporation, ${ }^{5}$ Department of \\ Mathematics, Statistics and Physics, \\ College of Arts and Sciences, Qatar \\ University, ${ }^{6}$ Neurology Department, \\ Weill Cornell Medicine - Qatar, \\ Doha, Qatar \\ *These authors contributed equally \\ to this work
}

Introduction: The elderly population is increasing around the world, and the prevalence of dementia increases with age. Hence, it is expected that the number of people with dementia will increase significantly in the coming years. The Mini-Mental Status Examination - 2 (MMSE-2) and Mini-Cog are widely used tests to screen for dementia. These scales have good reliability and validity and are easy to administer in clinical and research settings.

Aim: The purpose of this study was to validate the Arabic versions of MMSE-2 and Mini-Cog. These scales were assessed against the Diagnostic and Statistical Manual of Mental Disorders, Fourth Edition, Text Revision (DSM-IV-TR) criteria for dementia, as the gold standard.

Methods: The standard versions of the MMSE-2 and Mini-Cog were translated to Arabic following the back-translation method. Then, a trained rater administered these tests to 134 Arab elderly aged $>60$ years. A physician, blind to the results of these two tests, assessed the participants for vascular dementia or probable Alzheimer's disease, based on the DSM-IV-TR criteria.

Results: The sample included $67.2 \%$ Qataris. The mean age was 74.86 years (standard deviation $=7.71$ ), and $61.9 \%$ did not attend school. The mean of the adjusted scores of MMSE-2 based on age and education level was 19.60 (standard deviation $=6.58$ ). According to $D S M-I V-T R$, $17.2 \%$ of the participants had dementia. Sensitivity and specificity of the MMSE-2 and the Mini-Cog together were $71.4 \%$ and $61.6 \%$, respectively, which were better than those of each test alone.

Conclusion: Together, the Arabic versions of MMSE-2 and Mini-Cog are good screening tools for cognitive impairment in Arabs.

Keywords: dementia, validation, MMSE-2, Mini-Cog, Arabic version

\section{Introduction}

Dementia is characterized by gradual deterioration in cognition resulting in significant impairments in daily functioning. ${ }^{1}$ Dementia is diagnosed on clinical grounds, and despite advances in medicine and technology, no particular laboratory tests or neuroimaging studies can be used to make a diagnosis of dementing illnesses. The onset of dementia is mostly insidious, and the course of the illness is usually a progressive decline in higher cortical functions and daily life activities; the course of progression varies from subacute to chronic depending on the etiology. Dementia affects each person differently and also has a significant impact on families and caregivers. ${ }^{2}$ Early diagnosis of cognitive impairment may increase the chance of a slower progression of the disease. ${ }^{3}$ Early intervention can provide caregivers with early advice and support. ${ }^{4}$

Dementia screening tools should be brief, easy to use and valid in different cultures and across elderly with various educational backgrounds. ${ }^{5}$ The Mini-Mental Status Examination (MMSE) and the Mini-Cog have been demonstrated in several
Correspondence: Hassen Al-Amin Psychiatry Department, Weill Cornell Box 24144, Doha, Qatar Tel +97444928313

Fax +974 44928377

Email haa2019@qatar-med.cornell.edu (c)
hereby accept the Terms. Non-commercial uses of the work are permitted without any further permission from Dove Medical Press Limited, provided the work is properly attributed. For permission for commercial use of this work, please see paragraphs 4.2 and 5 of our Terms (https://www.dovepress.com/terms.php). 
studies as valid and brief tests that provide satisfactory screening of cognitive deficits and determine their severity at the time of evaluation. They can also serve as measuring tools of progression or improvement of cognition in cognitive illness. ${ }^{6,7}$ These tests were originally made in English and were translated and scientifically validated in other languages. ${ }^{8,9}$ Although a recent version of MMSE attempted to minimize the impact of the confounding factors, most screening measures are often skewed by factors such as language, culture and level of education. ${ }^{10}$ To maximize the sensitivity and specificity of MMSE, experts recommend combining MMSE with Clock-Drawing Test (CDT) or Mini-Cog. ${ }^{11}$ These tests have not been scientifically validated in Arabic, and in general, the Arab countries are still lacking the proper tools to screen for dementia.

We did not find any informative epidemiological studies on the prevalence of dementia in the Middle East and North African (MENA) countries. The experts working with the World Health Organization (WHO) estimated that the prevalence of dementia is $\sim 6 \%$ among adults aged $>60$ years in the MENA countries. ${ }^{12,13}$ A study assessing the prevalence of mental illness in the Qatari population $(n=1,660)$ reported that dementia accounted for only $1.1 \%$ of the total sample but reached up to $52 \%$ in people aged $>50$ years $(n=297) .{ }^{14}$

Qatar is a rapidly growing country with several ethnicities and nationalities. The last official census in 2010 conducted by the Qatar Statistics Authority ${ }^{15}$ showed that the total population in Qatar is $\sim 1.5$ million where $40 \%$ of them are Arabs. The senior citizens (aged $>60$ years) accounted for only $2 \%$ of the total population with the majority of them being males. The aim of this study was to validate the new Arabic versions of the MMSE-2, standard version (SV), and the Mini-Cog test in the Arab elderly population residing in Qatar. We also determined the sensitivity and specificity for different cutoffs of MMSE-2:SV and Mini-Cog in predicting the clinical diagnosis of dementia in the Arab elderly population in Qatar. The diagnosis is based on the criteria for dementia in Diagnostic and Statistical Manual of Mental Disorders, Fourth Edition, Text Revision (DSM-IV-TR). We hypothesized that using both scales together (MMSE-2:SV and Mini-Cog) would result in more accurate diagnosis of dementia compared to either one alone.

\section{Methods}

\section{Subjects}

Subjects were recruited from the elderly population who attended the primary health care centers (PHCCs) and the outpatient services at the Psycho-geriatrics, Medical
Geriatrics and Neurology departments of Hamad Medical Corporation (HMC), Doha, Qatar. In this study, the subjects recruited were only Arabic native speakers aged $>60$ years. The study excluded elderly who: 1) had depression or other psychiatric disorders other than dementia, 2) had a major medical or neurological disorder that mandated acute treatment or needed immediate care in the 4 weeks before enrollment, 3) used psychotropics or anti-dementia medications for more than 4 weeks before the recruitment time to avoid the confounding effects of medications, 4) reported a history of drug or alcohol use, and 5) having hearing or vision impairment or showed presence of slurred or incomprehensible speech. The latter were excluded as instructions had to be explained to the subjects and their answers involve copying and comprehensive verbal communication.

We do not have consensus on the number of elderly attending these different clinics, but between June 2013 and March 2014, the investigators screened $\sim 420$ elderly subjects where 276 subjects fulfilled the inclusion and exclusion criteria and of which only 134 accepted enrollment in the study. All of them completed the MMSE-2:SV and were clinically assessed if they met the criteria for dementia according to $D S M-I V-T R$, but only 113 had a conclusive Mini-Cog as the rest could not complete the CDT because of illiteracy (could not read or write the numbers). The sample size differed across validations of different scales. Figure 1 illustrates the number of subjects who finalized the different assessments along with the sample size for each analysis.

In regard to sample size, we believe that we satisfied the condition of having at least 10 measures for each item, ${ }^{16}$ as the MMSE-2 has 11 items and the additional one from Mini-Cog is the CDT. The sample was recruited using convenient sampling technique. The selected sample size was also adequate for the estimation of sensitivity and specificity to within a maximum margin of error of $7 \%$ at a $95 \%$ confidence interval (CI). It was also consistent with the number of patients used in the other studies that validated the translated scales in other languages such as Persian. ${ }^{17}$

The Institutional Review Board of HMC approved the study. It was granted an exemption from obtaining written informed consent, as all the tests administered are usually part of routine clinical screening for elderly patients. However, the participants (or a family member) were given a written document, which explained the purpose of the study, the tests to be done and the estimated time needed to finish them. 


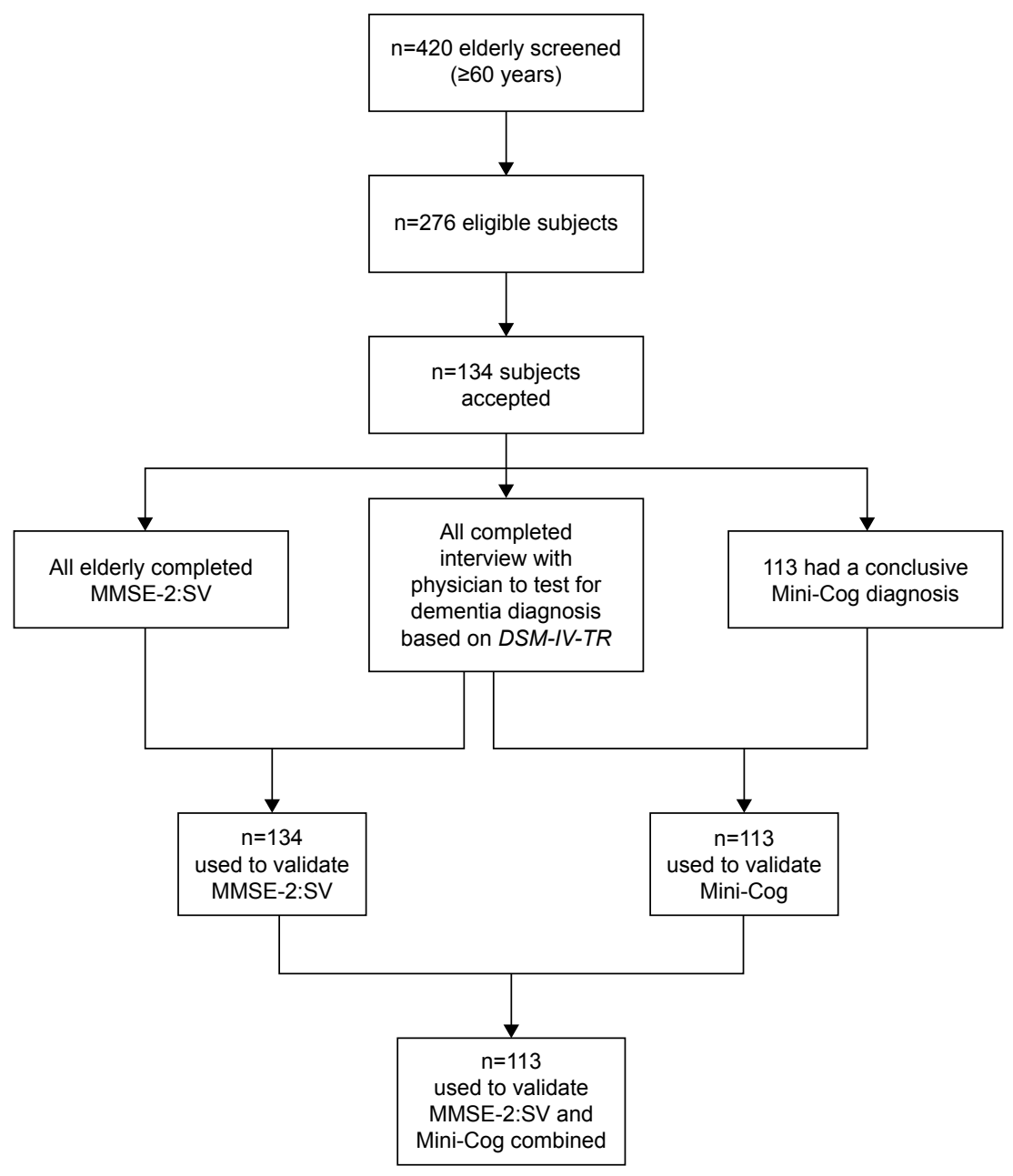

Figure I A flow chart showing the number of subjects and what tests they completed.

Abbreviations: MMSE-2, Mini-Mental Status Examination - 2; SV, standard version; DSM-IV-TR, Diagnostic and Statistical Manual of Mental Disorders, Fourth Edition, Text Revision.

\section{MMSE and MMSE-2}

The original MMSE ${ }^{18}$ is the most commonly used cognitive screening test worldwide. The examination takes $\sim-10$ minutes to complete. The total score is 30 , and it tests a broad range of cognitive functions, including orientation, recall, attention, calculation, language processing and constructional praxis. ${ }^{18}$ Previous studies have suggested that different cutoff scores on MMSE are needed to screen for cognitive impairment and control for the differences in age, education and cultural variations. ${ }^{19}$ In a study by Folstein and Folstein, ${ }^{20}$ the following cutoffs were determined: $27-30$ is normal, $21-26$ is mild impairment, $11-20$ is moderate impairment and $\leq 10$ is severe impairment. The most widely used cutoff to suggest dementia is a score of $<24 .{ }^{21}$ This cutoff score $<24$ yielded a sensitivity and specificity of $58 \%$ and $98 \%$, respectively. ${ }^{21}$ The SV of the MMSE-2 (MMSE-2:SV) is one of the three revised versions of MMSE. It retains the structure and scoring of the original 30-point MMSE, but the problematic items were replaced and several tasks were modified to minimize difficulty and to facilitate its translation into foreign languages. It has been tested in a normal population and in people with Alzheimer's disease and subcortical dementia. The raw score range (ie, $0-30$ ) remains the same, and the MMSE-2:SV and MMSE cutoffs are comparable. ${ }^{22}$

\section{Mini-Cog test}

Mini-Cog requires only 3 minutes to administer and consists of a CDT and non-cued recall of three unrelated words. Mini-Cog attained 99\% sensitivity and 93\%-96\% specificity in elderly from diverse ethnic and linguistic backgrounds where the prevalence of dementia was $50 \% .^{5,11}$ The advantages of the Mini-Cog include brevity, ease of administration, acceptability to patients, simple scoring and high sensitivity for predicting dementia status and diagnostic value not limited by the subject's education, language or cultural bias. Mini-Cog is more sensitive to mild dementia than MMSE. ${ }^{5}$ 
It is also useful for assessing visuoconstructive abilities, including praxis and executive higher functions.

\section{Translation and cultural adaptation}

After obtaining the proper permission from the copyright holders of the MMSE-2, ie, Psychological Assessment Resources (PAR), a committee of one professional translator, three bilingual psychiatrists and a neurologist independently translated the MMSE-2 and Mini-Cog. The committee deliberated over few sessions to decide anonymously on one preliminary Arabic version for each test. A pilot study was then carried out to test the language and clarity of the scales in a sample of Arab elderly $(n=20)$. The concerns of the subjects were about the different terms used in the section on orientation to place such as state vs country, city vs town, etc., but these can be changed to fit with the country of the participants as indicated in the English version. Other concerns were about the choice of words to remember, the serial sevens and the meaning of the sentence to repeat but not about the Arabic translation per se. However, with encouragement, subjects were able to perform these sections. Finally, they hesitated to write a sentence but then were able to do it with more prompting. In regards to the Mini-Cog, subjects needed more explanations on how to do the clock drawing and some added that they "do not know how to draw a clock". The physicians and nurses who administered the scales commented on the Arabic translations for the levels of consciousness where few mentioned that these terms are not commonly used by lay people, and it was explained that this is usually assessed by trained individuals and not by patients. The comments and issues were then raised to the committee, and further changes were made accordingly. The final version that was approved by the committee was then back translated to English by a new independent professional translator and was compared to the original version by the committee of psychiatrists. This back translation was sent back to PAR that reviewed and suggested making two changes before their approval of the final back translation. The two changes were related to two words (aloud and comprehension) where their Arabic translations gave different meanings in the back translation but the Arabic words were changed to fit linguistically with the original English translation.

\section{Procedure}

The psycho-geriatricians involved in the study trained two raters on the administration of MMSE-2 and were supervised on several cases before allowing them to finalize them alone. After obtaining the assent of the participant, a rater collected the sociodemographic data (such as age, gender, educational level, etc.) and the clinical history from the patient and/or his family. Medical records were also checked to retrieve the missing data. After that, the trained raters administered MMSE-2 and Mini-Cog tests. Then, a physician, who was blind to the scores on MMSE-2 and Mini-Cog, interviewed all the participants and assessed whether they clinically met the criteria for dementia (either Alzheimer's or vascular dementia or both) based on DSM-IV-TR. The physicians involved (one neurologist, three geriatricians, two psycho-geriatricians and one primary care physician) met before the start of recruitment, reviewed the $D S M-I V$ criteria and agreed on the definitions and the clinical assessment of the criteria.

\section{Statistical analysis}

The sample was described in terms of age, gender, country born and educational level for 134 cases (refer to Figure 1 for details on sample sizes for the different analyses). The sample was split into two groups based on whether the individuals met the criteria of either type of dementia (vascular or Alzheimer as per DSM-IV-TR) or not. The raw scores on MMSE-2 were corrected based on age and educational level of the participants; we also used the $T$-scores that are provided by PAR in the MMSE-2 Manual. Scores' range and mean were calculated. Adjusted scores were compared across males and females (using paired $t$-test) and different educational levels (using analysis of variance [ANOVA]) with Bonferroni corrections for multiple comparisons. Effect sizes were also reported. Pearson's correlation was carried out to test if age and MMSE-2 adjusted scores were correlated. The prevalence of dementia in the sample was calculated, and differences between females and males were tested using chi-square test.

Using receiver-operating characteristic (ROC) curves, separate analyses were carried out to determine the sensitivity and specificity of each of the MMSE-2:SV and Mini-Cog compared to the gold standard (clinical diagnosis based on $D S M-I V-T R$ ). After that, ROC curves were drawn to assess the sensitivity and specificity for using both scales together compared to those who have dementia diagnosis on both MMSE2:SV and Mini-Cog. Statistical analyses were carried out using IBM-Statistical Package 23.0 (IBM Corporation, Armonk, NY, USA). Statistical significance was set at 0.05 level.

\section{Results}

The sample ( $\mathrm{n}=134$ ) had more males than females, $61.9 \%$ and $38.1 \%$, respectively. The age group ranged between 60 and 96 years. The mean age was $74.86(\mathrm{SD}= \pm 7.71)$. In this 
sample, there were more people born in Qatar than in the other Arab countries (including Egypt, Iraq, Jordan, Lebanon, Libya, Morocco, Oman, Palestine, Saudi Arabia, Sudan, Syria and Yemen), 63.1\% and 36.9\%, respectively. More than half $(61.9 \%)$ of the sample did not attend school. Table 1 lists the frequencies and percentages of the sociodemographic variables based on whether patients were diagnosed with dementia (as per DSM-IV-TR criteria) or not. In this sample, there were 23 (12.3\%) subjects with dementia vs 111 without. In regard to dementia diagnosis as per DSM-IV-TR, 21 patients had vascular dementia and two cases had possible Alzheimer's dementia. There were no significant differences based on gender, age and educational level, but the Qataris were significantly more in both groups compared to other Arabs $(P<0.05)$.

Table 2 lists the various scores obtained in the two groups (dementia vs no dementia). The MMSE-2 and MiniCog scores in the dementia group were significantly lower compared to those without clinical dementia $(P<0.05$; Table 2). Based on the Mini-Cog scores, 78 elderly (58.2\%) were potentially having dementia out of the total sample that had conclusive Mini-Cog $(n=113)$. Only 61 subjects

Table I Sociodemographic data by dementia diagnosis according to DSM-IV-TR

\begin{tabular}{|c|c|c|}
\hline \multirow[t]{2}{*}{ Variable } & \multirow{2}{*}{$\frac{\text { Dementia }(\mathrm{n}=23)}{\text { Frequency }(\%)}$} & \multirow{2}{*}{$\frac{\text { No dementia }(\mathrm{n}=\mathrm{I} \text { I I })}{\text { Frequency }(\%)}$} \\
\hline & & \\
\hline \multicolumn{3}{|l|}{ Gender } \\
\hline Male & $12(52.2)$ & $7 \mid(64.0)$ \\
\hline Female & II (47.8) & $40(36.0)$ \\
\hline \multicolumn{3}{|l|}{ Age (years) } \\
\hline $60-64$ & $2(8.7)$ & 13 (II.7) \\
\hline $65-69$ & $0(0)$ & $18(16.2)$ \\
\hline 70-74 & $7(30.4)$ & $26(23.4)$ \\
\hline $75-79$ & $5(21.7)$ & $23(20.7)$ \\
\hline $80-84$ & $6(26.1)$ & $16(14.4)$ \\
\hline $85-89$ & $3(13.0)$ & $7(6.3)$ \\
\hline $90+$ & $0(0)$ & $4(3.6)$ \\
\hline Unknown & $0(0)$ & $4(3.6)$ \\
\hline \multicolumn{3}{|l|}{ Country born } \\
\hline Qatar* & $20(87.0)$ & $70(63.1)$ \\
\hline Other & $3(13.0)$ & $40(36.0)$ \\
\hline Unknown & $0(0)$ & I (0.9) \\
\hline \multicolumn{3}{|l|}{ Educational level } \\
\hline No schooling & I8 (8I.8) & $65(58.6)$ \\
\hline Intermediate & $2(9.1)$ & II (9.9) \\
\hline Secondary & $0(0)$ & $10(9.0)$ \\
\hline College and above & $2(9.1)$ & $15(13.5)$ \\
\hline Unknown & I (4.3) & $10(9.0)$ \\
\hline
\end{tabular}

Note: $* P<0.05$, more Qatari in both groups compared to other Arabs.

Abbreviation: DSM-IV-TR, Diagnostic and Statistical Manual of Mental Disorders, Fourth Edition, Text Revision.
Table 2 Mean (SD) scores on Arabic versions of MMSE-2:SV and Mini-Cog by dementia diagnosis (using DSM-IV-TR criteria) in the study sample

\begin{tabular}{|c|c|c|}
\hline \multirow[t]{2}{*}{ Scales } & $\begin{array}{l}\text { Dementia } \\
(n=23)\end{array}$ & $\begin{array}{l}\text { No dementia } \\
(\mathrm{n}=\text { IIII) }\end{array}$ \\
\hline & $\overline{\text { Mean (SD) }}$ & Mean (SD) \\
\hline \multicolumn{3}{|l|}{ MMSE-2:SV $(n=134)$} \\
\hline Raw scores* & $15.26(4.06)$ & $19.52(7.12)$ \\
\hline$T$-scores* & $21.41(13.47)$ & $32.57(18.68)$ \\
\hline $\begin{array}{l}\text { Adjusted scores* (for age } \\
\text { and level of education) }\end{array}$ & $16.39(4.21)$ & $20.42(6.82)$ \\
\hline \multicolumn{3}{|l|}{ Mini-Cog $(n=|| 3)$} \\
\hline Score* & $1.55(0.89)$ & $2.07(0.47)$ \\
\hline
\end{tabular}

Note: *Scores are lower in the dementia group, $P<0.05$.

Abbreviations: MMSE-2, Mini-Mental Status Examination -2; SD, standard deviation; SV, standard version; DSM-IV-TR, Diagnostic and Statistical Manual of Mental Disorders, Fourth Edition, Text Revision.

completed the CDT, and in 32 elderly (52.5\%), the drawing was incorrect.

The results also showed that there was no significant difference between males and females on the Mini-Cog score $(P=0.180)$. However, there were differences in MMSE-2:SV adjusted scores, $P=0.004$. Males scored higher on MMSE-2:SV adjusted scores compared to females, with mean values $21.11(\mathrm{SD}=6.48)$ and $17.16(\mathrm{SD}=6.03)$, respectively. There was a significant negative correlation between the participants' age and their adjusted scores on MMSE-2:SV, $r=-0.46$ and $P<0.01$. This correlation has medium to large effect size.

MMSE-2 mean-adjusted score was higher in groups of elderly with higher levels of education compared to those with no education. The mean-adjusted scores on MMSE-2 for educational levels no schooling, intermediate schooling, secondary schooling and college and above were 16.66 ( $\mathrm{SD}=5.24), 23.00$ ( $\mathrm{SD}=4.69), 25.60$ ( $\mathrm{SD}=5.15)$ and 26.53 $(\mathrm{SD}=4.88)$, respectively. ANOVA showed a significant difference in MMSE-2:SV scores across elderly with different levels of education, $F(3,119)=25.83, P=0.001$ and partial eta-squared $\eta^{2}=0.39$ for the differences between groups. Bonferroni post hoc tests showed only significant differences on MMSE-2:SV adjusted scores between no schooling and each of the other three groups, $P<0.001$.

Similarly, there were significant differences on the MiniCog scores between elderly with different levels of education, $F(3,98)=10.91, P<0.01$ and partial eta-squared $\eta^{2}=0.25$. However, post hoc analysis showed significant differences between no schooling and attended high school (mean difference $=0.95$, standard error $[\mathrm{SE}]=0.27, P=0.003, \mathrm{CI}$ $[0.24,1.67])$ and between those with no schooling and those 
Table 3 Sensitivity and specificity of the Arabic versions of MMSE-2:SV and Mini-Cog compared to clinical diagnosis of dementia using DSM-IV-TR criteria

\begin{tabular}{|c|c|c|c|c|c|c|c|c|}
\hline \multirow[t]{2}{*}{ Variables } & \multirow[t]{2}{*}{$\mathbf{n}$} & \multirow[t]{2}{*}{ AUC } & \multirow[t]{2}{*}{ SE } & \multirow[t]{2}{*}{ Cutoff } & \multicolumn{2}{|l|}{$\mathrm{Cl}$} & \multicolumn{2}{|l|}{ Accuracy } \\
\hline & & & & & Lower bound & Upper bound & Sensitivity (\%) & Specificity (\%) \\
\hline MMSE-2:SV adjusted scores & 134 & $0.68^{*}$ & 0.05 & $18 / 19$ & 0.580 & $0.78 \mathrm{I}$ & 60.9 & 59.5 \\
\hline MMSE-2:SV T-scores & 134 & $0.66^{*}$ & 0.06 & $21 / 22$ & 0.550 & 0.778 & 59.1 & 68.7 \\
\hline Mini-Cog score & 113 & 0.65 & 0.07 & 1.5 & 0.517 & 0.777 & 92.9 & 34.3 \\
\hline MMSE-2:SV and Mini-Cog & 113 & $0.65^{*}$ & 0.08 & $20 / 21$ & 0.516 & 0.814 & 71.4 & 61.6 \\
\hline
\end{tabular}

Note: $* P<0.05$.

Abbreviations: MMSE-2, Mini-Mental Status Examination - 2; SV, standard version; DSM-IV-TR, Diagnostic and Statistical Manual of Mental Disorders, Fourth Edition, Text Revision; AUC, area under the curve; SE, standard error; $\mathrm{Cl}$, confidence interval.

who attended college and above (mean difference $=1.01$, $\mathrm{SE}=0.20, P<0.001$, CI $[0.46,1.56])$.

The results of the ROC curves for the different scales are listed in Table 3. For the MMSE-2:SV adjusted scores, the sensitivity and specificity were $60.9 \%$ and $59.5 \%$ with an area of $0.68, P<0.05$. The results showed that the best cutoff was 18/19 for these scores. According to this cutoff, 59 elderly had dementia (44\% of the sample of 134 subjects). Figure 2 illustrates the ROC curves for the individual and combined scores. The $T$-scores showed better specificity than the adjusted scores but with a lesser sensitivity. Mini-Cog did not have a significant area under the curve. However, its
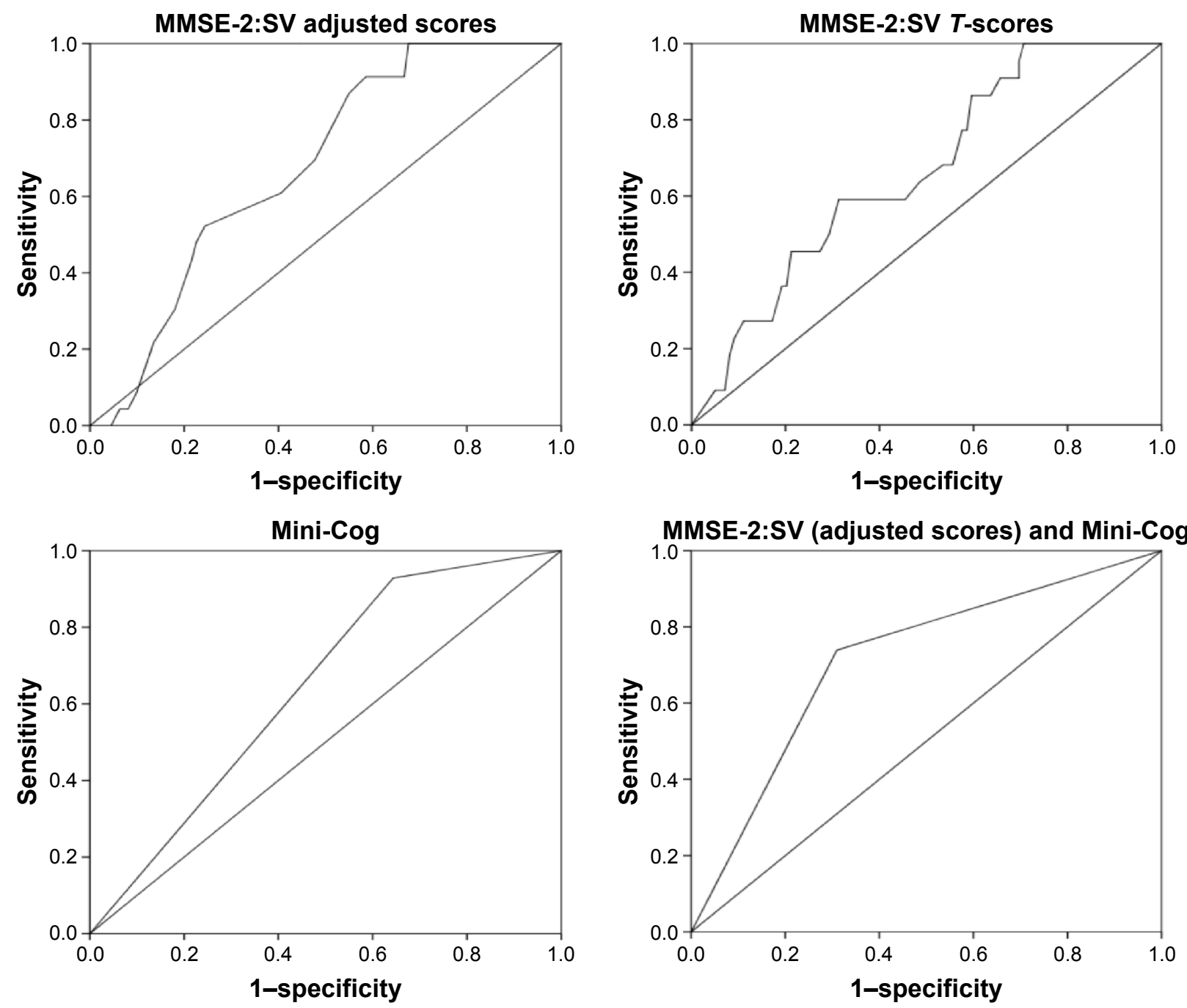

Figure 2 ROC curves for the unique and combined scores of the Arabic version of MMSE-2:SV and Mini-Cog compared to diagnosis of dementia according to DSM-IV-TR criteria. Notes: The combined scores of MMSE-2:SV (adjusted) and Mini-Cog produced the optimal sensitivity (7I.4\%) and specificity (6I.6\%) where the AUC was 0.65 ( $P<0.05)$ with SE of 0.08 and $\mathrm{Cl}$ of $0.516-0.814$. The cutoff for the combined scores were 20/2I.

Abbreviations: ROC, receiver-operating characteristic; MMSE-2, Mini-Mental Status Examination - 2; SV, standard version; DSM-IV-TR, Diagnostic and Statistical Manual of Mental Disorders, Fourth Edition, Text Revision; AUC, area under the curve; Cl, confidence interval; SE, standard error. 
sensitivity was excellent $(92.9 \%)$, but the specificity was very low (34.3\%). Combining these two scales together, Mini-Cog and MMSE-2:SV adjusted scores added to the sensitivity and specificity of MMSE-2:SV adjusted scores, 74.4\% and $61.6 \%$, respectively. Based on MMSE-2:SV and Mini-Cog scores, 48 elderly had dementia, making up to $42.5 \%$ of the sample studied $(\mathrm{n}=113)$.

\section{Discussion}

The aim of this study was to translate and validate the Arabic versions of the MMSE-2:SV and Mini-Cog test. Compared to the clinical diagnosis according to $D S M-I V-T R$ criteria, the combined Arabic versions of MMSE-2:SV and Mini-Cog were valid screening tools for dementia. Our results showed that screening for dementia in the Arab population, the Arabic MMSE-2:SV with the Arabic Mini-Cog, improved the balance between sensitivity and specificity than using either measure alone. This is the first study to translate, culturally adapt and scientifically validate the Arabic versions of these widely used tools to screen for cognitive impairment in the elderly. The official language used in all Arab countries is formal Arabic. Hence, we translated the above tools to formal Arabic so that they could be used across the different Arab countries. Other strengths of this study are the standard procedures for translation and validation, adjustment of MMSE-2 scores by age and education and proper training of the raters who were blinded to the diagnosis that was performed by qualified clinicians and according to the standard criteria of $D S M-I V-T R$. Recently and after the start of this study, two other studies were published on the validation of the Arabic version of the 10/66 Dementia Research Group (DRG) diagnostic assessment for dementia ${ }^{23}$ and the Arabic Rowland Universal Dementia Assessment Scale (A-RUDAS) ${ }^{24}$ in the elderly population of Lebanon. These studies reported that these scales are better suited for assessment of dementia in the Arab population where the illiteracy is very high. However, we do believe that the brevity and ease of administration of the MMSE-2 and Mini-Cog and familiarity of health care providers with both tests are important factors in the facilitation of screening for cognitive impairment in epidemiological studies and in routine clinics and wards.

The Arabic version of the MMSE-2:SV demonstrated good specificity but low sensitivity. The cutoff in this sample was $18 / 19$, which is similar to that found in other cultures. ${ }^{25,26}$ Nevertheless, this cutoff is less than what is usually found across different studies. ${ }^{21,27}$ Age and education are known confounders of the results of MMSE, ${ }^{19}$ which were different in this sample compared to other studies, as the majority of the subjects enrolled in this study were illiterate and $>70$ years. Caramelli et $\mathrm{al}^{28}$ showed that a score of 18 on MMSE is the best cutoff for illiterate elderly, which supports the validity of our results in the Arab population where the illiteracy is high in the elderly population.

The Arabic version of the Mini-Cog showed good sensitivity and low specificity. This brief assessment did not accurately predict a diagnosis of dementia. In the MMSE-2, the raw scores could be adjusted based on age and education. However, in the Mini-Cog, the results remained inconclusive and these individuals were dropped from the main analysis, which might underpower the results of the Arabic Mini-Cog. However, when used with the Arabic version of MMSE-2:SV, the sensitivity and specificity were good and the significant measures were considered valid ones to test for dementia in elderly Arabs. Other studies also supported the use of Mini-Cog with MMSE in patients with cognitive deficits for more accurate screening outcomes. ${ }^{11}$

\section{Limitations}

This study has several strengths that were mentioned earlier, but it also has few limitations that are worth discussing. First, a good number of elderly could not complete several items on MMSE-2 and Mini-Cog due to their inability to read, write and draw. This could affect the power of our results. Thus, future studies with larger samples are needed to control for this issue. Second, although the study included Arabs from different countries, the majority of Arabs in this sample were born in Qatar, and thus larger studies with better representation of the different Arab countries can also control for the effects of subcultures in the different Arab countries. Third, there could be a gender effect in this study as we had more males in the enrolled sample, which might limit the generalizability of our results across gender. Fourth, MMSE is not sensitive to detect mild dementia, and scores may be influenced by age, education level, cultural background, social class, literacy and language. ${ }^{19,29-31}$ We tried to control for many of these factors, but the gold standard used to test the validity of Arabic MMSE-2:SV and Mini-Cog does not provide a clear assessment of the severity of dementia. Thus, we could not use the scores of Arabic MMSE-2 to assess the various degrees of cognitive impairment.

\section{Conclusion}

The Arabic versions of the MMSE-2 and Mini-Cog are valid tools when used together for screening dementia. This study provides the foundation for future work on elderly with cognitive deficits in the Arab world. Future work might need to focus on creating versions that adapt to the various educational levels in various Arabic subcultures. The adaptation of these 
scales to Arabic-speaking communities will also make it possible to explore with more detail the epidemiology of dementia and facilitate comparisons with international studies in regard to risk factors, prognosis and treatment.

\section{Acknowledgments}

We would like to thank Dr Essa Al-Sulaiti for his valuable support in facilitating the recruitment of subjects and for his input on the manuscript. This study was funded to M Albanna and H Al-Amin as a research grant from Medical Director's Grant Competition of HMC in Doha, Qatar (grant number: GC 1011A). The funding agency had no role in the design, implementation or interpretation of the results of this study.

\section{Author contributions}

$\mathrm{H}$ Al-Amin and M Albanna designed the study and supervised data collection. $\mathrm{H} \mathrm{Al}$-Amin finalized the data analysis and the writing of the article. M Albanna and A Yehya contributed equally to preparing the manuscript. A Yehya and A Yousif were responsible for carrying out the statistical analysis. A Khairi, E Dafeeah, A Elhadi, L Rezgui, S Al Kahlout and B Uthman collected the data and assisted with writing the article. All authors contributed toward data analysis, drafting and critically revising the paper and agree to be accountable for all aspects of the work.

\section{Disclosure}

The authors report no conflicts of interest in this work.

\section{References}

1. APA. Diagnostic and Statistical Manual of Mental Disorders. 5th ed. Washington, DC: American Psychiatric Association; 2013.

2. Connell CM, Boise L, Stuckey JC, Holmes SB, Hudson ML. Attitudes toward the diagnosis and disclosure of dementia among family caregivers and primary care physicians. Gerontologist. 2004;44(4):500-507.

3. Leifer BP. Early diagnosis of Alzheimer's disease: clinical and economic benefits. J Am Geriatr Soc. 2003;51(5 suppl Dementia):S281-S288.

4. de Vugt ME, Verhey FR. The impact of early dementia diagnosis and intervention on informal caregivers. Prog Neurobiol. 2013;110:54-62.

5. Lorentz WJ, Scanlan JM, Borson S. Brief screening tests for dementia. Can J Psychiatry. 2002;47(8):723-733.

6. Mitchell AJ. A meta-analysis of the accuracy of the Mini-Mental State Examination in the detection of dementia and mild cognitive impairment. J Psychiatr Res. 2009;43(4):411-431.

7. Tsoi KK, Chan JY, Hirai HW, Wong SY, Kwok TC. Cognitive tests to detect dementia: a systematic review and meta-analysis. JAMA Intern Med. 2015;175(9):1450-1458.

8. Steis MR, Schrauf RW. A review of translations and adaptations of the Mini-Mental State Examination in languages other than English and Spanish. Res Gerontol Nurs. 2009;2(3):214-224.

9. Milian M, Leiherr AM, Straten G, Muller S, Leyhe T, Eschweiler GW. The Mini-Cog versus the Mini-Mental State Examination and the Clock Drawing Test in daily clinical practice: screening value in a German Memory Clinic. Int Psychogeriatr. 2012;24(5):766-774.
10. Woodford HJ, George J. Cognitive assessment in the elderly: a review of clinical methods. QJM. 2007;100(8):469-484.

11. Scanlan J, Borson S. The Mini-Cog: receiver operating characteristics with expert and naive raters. Int J Geriatr Psychiatry. 2001;16(2): 216-222.

12. Ferri CP, Prince M, Brayne C, et al. Global prevalence of dementia: a Delphi consensus study. Lancet. 2005;366(9503):2112-2117.

13. Prince M, Bryce R, Albanese E, Wimo A, Ribeiro W, Ferri CP. The global prevalence of dementia: a systematic review and meta-analysis. Alzheimers Dement. 2013;9(1):63e62-75e62.

14. Ghuloum S, Bener A, Abou-Saleh MT. Prevalence of mental disorders in adult population attending primary health care setting in Qatari population. J Pak Med Assoc. 2011;61(3):216-221.

15. Census 2010 [homepage on the internet]. Qatar Statistical Authority; (cited 2014 July 15). Available from: www.qsa.gov.qa. Accessed January 26, 2016.

16. Nunnally J. Psychometric Theory. New York, NY: McGraw Hill; 1978.

17. Ansari NN, Naghdi S, Hasson S, Valizadeh L, Jalaie S. Validation of a Mini-Mental State Examination (MMSE) for the Persian population: a pilot study. Appl Neuropsychol. 2010;17(3):190-195.

18. Folstein MF, Folstein SE, McHugh PR. "Mini-mental state". A practical method for grading the cognitive state of patients for the clinician. J Psychiatr Res. 1975;12(3):189-198.

19. Lopez MN, Charter RA, Mostafavi B, Nibut LP, Smith WE. Psychometric properties of the Folstein Mini-Mental State Examination. Assessment. 2005;12(2):137-144.

20. Folstein MF, Folstein SE. Mini Mental State Examination (MMSE): User Manual. 2nd ed. Lutz, FL: Psychological Assessment Resources; 2001.

21. Folstein MF, Folstein SE, Fanjiang G. MMSE Mini-Mental State Examination: Clinical Guide. Lutz, FL: Psychological Assessment Resources; 2001.

22. Folstein MF, Folstein SE, McHugh PR, Fanjiang G. MMSE Examen Cognoscitivo Mini-Mental. Madrid: TEA Ediciones; 2002.

23. Phung KT, Chaaya M, Waldemar G, et al. Validation of the $10 / 66$ Dementia Research Group diagnostic assessment for dementia in Arabic: a study in Lebanon. J Geriatr Psychiatry Neurol. 2014;27(4): 282-290.

24. Chaaya M, Phung TK, El Asmar K, et al. Validation of the Arabic Rowland Universal Dementia Assessment Scale (A-RUDAS) in elderly with mild and moderate dementia. Aging Ment Health. 2016; 20(8):880-887.

25. Bertolucci PH, Brucki SM, Campacci SR, Juliano Y. O Mini-Exame do Estado Mental em uma população geral. Impacto da escolaridade [The Mini-Mental State Examination in a general population: impact of educational status]. Arq Neuropsiquiatr. 1994;52(1):1-7. Portuguese.

26. Lourenco RA, Veras RP. Mini-Exame do Estado Mental: características psicométricas em idosos ambulatoriais [Mini-Mental State Examination: psychometric characteristics in elderly outpatients]. Rev Saude Publica. 2006;40(4):712-719. Portuguese.

27. Spering CC, Hobson V, Lucas JA, Menon CV, Hall JR, O'Bryant SE. Diagnostic accuracy of the MMSE in detecting probable and possible Alzheimer's disease in ethnically diverse highly educated individuals: an analysis of the NACC database. J Gerontol A Biol Sci Med Sci. 2012; 67(8):890-896.

28. Caramelli P, Poissant A, Gauthier S, et al. Educational level and neuropsychological heterogeneity in dementia of the Alzheimer type. Alzheimer Dis Assoc Disord. 1997;11(1):9-15.

29. Jorm AF, Scott R, Henderson AS, Kay DW. Educational level differences on the Mini-Mental State: the role of test bias. Psychol Med. 1988; 18(3):727-731.

30. Liu HC, Teng EL, Lin KN, et al. Performance on a dementia screening test in relation to demographic variables. Study of 5297 community residents in Taiwan. Arch Neurol. 1994;51(9):910-915.

31. Freidl W, Schmidt R, Stronegger WJ, Irmler A, Reinhart B, Koch M. Mini mental state examination: influence of sociodemographic, environmental and behavioral factors and vascular risk factors. $J$ Clin Epidemiol. 1996;49(1):73-78. 


\section{Publish your work in this journal}

Neuropsychiatric Disease and Treatment is an international, peerreviewed journal of clinical therapeutics and pharmacology focusing on concise rapid reporting of clinical or pre-clinical studies on a range of neuropsychiatric and neurological disorders. This journal is indexed on PubMed Central, the 'PsycINFO' database and CAS, and is the official journal of The International Neuropsychiatric Association (INA). The manuscript management system is completely online and includes a very quick and fair peer-review system, which is all easy to use. Visit http://www.dovepress.com/testimonials.php to read real quotes from published authors.

\footnotetext{
Submit your manuscript here: http://www.dovepress.com/neuropsychiatric-disease-and-treatment-journal
} 